\title{
Efeitos da frequência de feedback na aprendizagem do saque do voleibol
}

\author{
Ivan W. Tertuliano ${ }^{1}$ \\ Alessandra A.C. U grinowitsch 2 \\ Herbert U grinowitsch 2 \\ U mberto C. Corrêa ${ }^{1}$
}

https://doi.org/10.5628/rpcd.07.03.328

\author{
1 Laboratório de Comportamento M otor \\ Escola de Educação Física e Esporte \\ Universidade de São Paulo \\ Brasil \\ ${ }^{2}$ Escola de Educação Física, \\ Fisioterapia e Terapia 0 cupacional \\ Universidade Federal de M inas G erais \\ Brasil
}

\begin{abstract}
RESUMO
Participaram deste estudo 20 crianças com idade variando entre oito e onze anos. A tarefa utilizada foi o saque por baixo do voleibol realizado da linha de ataque de uma quadra de voleibol, com o objectivo de acertar um alvo posicionado na linha de ataque da quadra oposta, o qual não podia ser visualizado pelos sujeitos. Foram formados dois grupos que diferiram em função da frequência feedback fornecida: uma informação a cada duas execuções (G50\%) ou uma informação a cada três execuções (G33\%). O experimento constou de 70 execuções na fase de aquisição e 10 execuções na fase de transferência. A segunda fase ocorreu 5 minutos após o término da aquisição, sem feedback. Os resultados mostraram o melhor desempenho do G33\% na fase de aquisição quando comparado ao G50\%, e, também na fase de transferência. Esses resultados indicam que a menor frequência de feedback auxilia na melhora do desempenho do saque por baixo do voleibol.
\end{abstract}

Palavras-chave: aprendizagem, frequência, feedback, voleibol.
ABSTRACT

Effects of the extrínsic feedback frequency in serve of volleyball learning

Twenty infants participated of this study aged from eight to eleven years. The task involved the volleyball under serve from the attack line in one side of the volleyball court aiming to reach one target positioned in the attack line of the opposite court, which could not be seen by the participants. There were two groups that deferred as a function of the frequency of augmented feedback: one information every two trials ( $G 50 \%$ ) or one information every three trials (G 33\%). The experiment had 70 trials in the acquisition phase and 10 trials in the transfer phase. The second phase was carried out 5 minutes after the last of the acquisition, without augmented feedback. The results showed the best performance of the $\mathrm{G} 33 \%$ in relation to $\mathrm{G} 50 \%$ during the acquisition phase and transfer test as well. These results indicate that to smaller frequency of feedback is helpful to improve the performance of the volleyball under serve.

Key-words: learning, frequency, feedback, volleyball. 


\section{INTRODUÇÃO}

O feedback é caracterizado como uma informação sensorial que indica algo sobre o estado real do movimento de uma pessoa(13). Segundo Tani(14), ele também pode ser caracterizado como uma informação sobre as situações passadas que podem ser utilizadas para controlar situações futuras, e assim alcançar o objectivo da tarefa. O feedback fornecido ao aprendiz pode conter informação relacionada ao: Conhecimento de Performance (CP) ou Conhecimento de Resultado (CR). O CP é informação verbalizada ou não verbalizada sobre a natureza do padrão de execução, apresentada após a execução de uma tarefa, responsável pelo resultado do movimento, frequentemente utilizado por instrutores e terapeutas em situações do dia-a-dia. O CR é a informação sobre o resultado da resposta no meio ambiente, apresentada após a execução de uma tarefa(7). A pesar de que, por definição, as duas formas de informação serem de fontes distintas, algumas pesquisas mostram que elas têm as funções compatíveis no que se refere à aprendizagem motora(20), ou seja, os mecanismos de CR e CP são os mesmos, o que difere são os diferentes aspectos da resposta que a informação se refere.

Apesar de ambos servirem como uma referência para execuções futuras, o CR é mais utilizado em pesquisas, pois é uma variável mais fácil de ser controlada, o que fornece maior fidedignidade aos resultados obtidos. Vários estudos têm testado diferentes formas de controlar a quantidade de informação fornecida. Uma delas, e a mais utilizada, é a frequência de $C R$, que se refere ao número de $C R s$ fornecidos em uma sequência de tentativas(6). Existem duas medidas diferentes de frequência: a relativa, que se refere à porcentagem de tentativas em que o CR é provido; a absoluta, que se refere ao número total de CRs fornecidos durante as tentativas(6). Uma outra forma de controlar a quantidade de informação fornecida é o feedback sumário, que é a informação sobre um conjunto de tentativas( ${ }^{(6)}$. Uma outra forma de controlar a quantidade de informação fornecida é denominada de amplitude de feedback. Nessa forma o feedback é fornecido só quando o aprendiz excede uma faixa pré-determinada, por exemplo, $10 \%$ de desvio do objectivo da tarefa(8).
Até a metade da década de 1970 acreditava-se que quanto mais preciso, frequente e imediato fosse $o$ $\mathrm{CR}$, mais visíveis seriam os seus efeitos na aprendizagem de habilidades motoras(3). Isso acontecia porque nos estudos não era realizado o teste de retenção ou de transferência, que diferenciam os efeitos transitórios do desempenho dos efeitos relativamente permanentes da aprendizagem.

Baird e Hughes(2), utilizando um del ineamento simiIar ao de Bilodeau e Bilodeau(3), real izaram um teste de retenção após a fase de prática, e os resultados mostraram uma tendência dos sujeitos com menor frequência relativa de CR terem desempenho superior aos sujeitos com maior frequência. Outros estu$\operatorname{dos}(1,5,20,17,21,23)$ corroboram esses resultados, indicando que frequências menores de CR são melhores para a aprendizagem, especificamente quando são aplicados os testes de transferência e retenção. Apesar desses estudos investigarem o efeito do CR na aquisição de habilidades motoras, eles não tinham a preocupação com "o que" era aprendido quando o desempenho apresentava melhora, ou seja, quando se tornava mais consistente. Para conseguir explicar isso, tradicionalmente os estudos estão recorrendo à Teoria de Esquema Motor(11), que propõe a aquisição de um programa motor generalizado (PMG) para uma classe de movimentos, que é uma representação armazenada na memória, ao qual são adicionados os parâmetros em cada execução.

Um dos estudos pioneiros que procurou investigar o efeito de frequência relativa de $C R$ na aprendizagem do PMG foi o de Wulf e Schmidt(23). Neste estudo, ambos os grupos realizaram o mesmo número de tentativas de prática durante a fase de aquisição, em um arranjo de prática em blocos de seis tentativas de modo seriado, num total de 108 tentativas para ambos os grupos. O grupo que praticou com $67 \%$ de frequência relativa de $C R$ obteve melhores resultados na fase de transferência que o grupo que praticou com $100 \%$ de CR. Resultados similares foram encontrados em outros estudos(22, 5).

Já estudos como os de Wulf, Lee e Schmidt(24) utilizaram quatro grupos com frequência de $50 \%$ e $100 \%$ de CR, em relação ao timing relativo (PMG) ou ao tempo absoluto (parâmetro) durante a fase de prática. Os resultados mostraram que, nos testes de retenção e transferência, a frequência reduzida auxi- 
liou a aprendizagem do PMG, mas não foi efectiva ou mesmo dificultou a aprendizagem de parâmetros. Chiviacowsky e Tani(6) também investigaram a frequência rel ativa de CR na aprendizagem de diferentes PMG. Os indivíduos tinham por objectivo acertar um botão de futebol de mesa em um alvo, sendo que a impulsão do botão foi realizada de três maneiras diferentes. Os grupos foram divididos em $50 \%$ e $100 \%$ de CR. Na fase de aquisição, os grupos apresentaram desempenho muito semelhante, porém, os testes de transferência mostraram uma superioridade do grupo de $50 \%$ de CR.

Ugrinowitsch, Tertuliano, Coca, Pereira e Gimenez ${ }^{(19)}$ investigaram os efeitos da frequência absoluta de feedback na aprendizagem de uma tarefa de preensão, na qual era requerida a aprendizagem de um novo parâmetro de força, utilizando um dinamómetro digital da Takey Instruments. Participaram do experimento 45 universitários, sem conhecimentos prévios da tarefa, os quais deveriam fazer uma preensão manual com $60 \%$ da força máxima, utilizando a mão não-dominante em um dinamómetro digital. Os sujeitos foram divididos em três grupos em relação ao fornecimento de feedback: G1, que recebeu feedback após cada execução; G2, que recebeu feedback a cada duas execuções e o G3, que recebeu feedback a cada três execuções. Os resultados mostraram que na primeira fase do experimento $o$ grupo G1 apresentou um melhor desempenho e diminuição da variabilidade, em contra partida, o grupo G3 foi o que apresentou maior consistência quando a meta da tarefa mudou para $40 \%$ da força máxima. Esses resultados permitem concluir que a menor frequência de feedback não é um factor prejudicial para a aprendizagem.

Tani, Meira Jr. e Gomes(16) investigaram o efeito de CR no processo adaptativo na aquisição de uma habilidade motora de controle de força manual, considerando as variáveis: precisão, frequência e localização temporal de CR. Em um dos experimentos o objectivo foi controlar a força em um dinamómetro, utilizando $50 \%$ da força máxima na fase de estabilização e $30 \%$ na fase de adaptação. Participaram do experimento 80 universitários, sem experiência pré via da tarefa. Os sujeitos foram divididos randomicamente em quatro grupos (G100, G66, G33, G20). $\mathrm{Na}$ fase de estabilização ( $50 \%$ da força máxima) , a frequência absoluta foi de dezoito (18) CRs para todos os grupos, com frequência relativa de $100 \%$, $66 \%$, 33\% e $20 \%$, respectivamente, para G100, G66, G33 e G20, cabendo para estes mesmos grupos, portanto, um total de 20, 29, 58 e 96 tentativas a executar. Para todos os grupos, a informação de CR contemplou magnitude e direcção do erro. $\mathrm{Na}$ fase de adaptação ( $30 \%$ de força máxima), todos os sujeitos executaram 10 tentativas sem o fornecimento de CR. Os resultados mostraram que na estabilização, G66 errou mais que o G100, e este mais que o G33 e G20. Na adaptação foi detectado que o G66 obteve pior performance em relação aos demais. Os autores concluíram que maior ou menor frequência de CR têm o mesmo efeito na aprendizagem de habilidades motoras.

Em suma, os primeiros estudos de feedback concluíram que as tentativas sem feedback não auxiliavam a aprendizagem. Posteriormente, com a incorporação dos testes de retenção e transferência, os resultados têm mostrado que a menor frequência facilita a aprendizagem. Tais resultados corroboram as conclusões de Ugrinowistch, Tertuliano, Coca, Pereira e Gimenez (19) e Weeks e Kordus(20) de que ocorre a aprendizagem mesmo com uma menor frequência de feedback.

Esses achados contradizem as conclusões dos primeiros estudos de que prover mais feedback é melhor para aprendizagem. Nessa nova visão, um número menor de CR é considerado necessário para que a aprendizagem ocorra, pois se essa quantidade for excessiva, os sujeitos tendem a não desenvolver os mecanismos internos de detecção e correcção de erros. Os resultados dos estudos revisados mostram que as frequências de feedback ficaram em torno de $25 \%$ a $66 \%$, porém não dizendo ao certo qual seria a melhor, e sim só que elas são melhores que os grupos controles ( $100 \%$ ). Nesse caso, ainda se faz necessário investigar qual a melhor faixa de frequência de CR a ser utilizada na aquisição de habilidades motoras.

O objectivo deste estudo foi investigar o efeito de duas frequências de feedback inferiores a 100\%, utilizando uma tarefa que privilegia a validade ecológica, já que todos os estudos supracitados utilizaram tarefas laboratoriais, nos quais há uma perda da validade ecológica. Nesse tipo de pesquisa busca-se colocar 
em prática a proposta de Chalmers(4), de testar os conhecimentos advindos da pesquisa básica em situações mais próximas do real. As pesquisas desse tipo valorizam a tarefa utilizada, tarefa do dia-a-dia do profissional, mas ainda buscando um grande controle das variáveis. Porém, como nesse tipo de tarefa há um certo "relaxamento" no controle das variáveis(18), como das variáveis intervenientes, os resultados ganham em validade ecológica(15). Seguindo esse raciocínio, é possível assumir que as pesquisas desenvolvidas dentro dessa linha podem fornecer maiores subsídios para os profissionais da área. Com base na hipótese de orientação(10), a qual assume que $C R$ frequente pode levar ao bloqueio do processamento das informações intrínsecas sobre o movimento anteriormente executado, que auxiliam o desenvolvimento do mecanismo de detecção e correcção de erros, fazendo com que o aprendiz se torne dependente da informação fornecida por meio de al gum meio externo, é possível especular que os resultados apresentem indicativos de superioridade para o grupo que receber menos informação externa.

\section{MÉTODO}

Participaram deste estudo 20 indivíduos de ambos os sexos, com idade entre 8 (oito) e 11 (onze) anos, participantes da Escola de Esportes do Clube da Cidade Vila Manchester. Os indivíduos não possuíam conhecimento prévio da tarefa praticada e participaram de forma voluntária, com seus pais assinando o termo de autorização.

A tarefa consistiu em realizar o saque por baixo de voleibol a partir do lado I da quadra (na região atrás da linha dos três metros) por sobre a rede, sem tocála, em direção a um alvo, visando al cançar a maior pontuação possível numa situação (teste adaptado de Teixeira(18)). A rede foi coberta com um plástico preto de forma que os indivíduos não tivessem a visão do alvo, e assim poder ser manipulada a variável dependente. $\mathrm{O}$ alvo foi colocado num plano horizontal no lado II (oposto ao lado de saque) da quadra de voleibol, na forma de um quadrado em um plástico azul, com as zonas de pontuação demarcadas por tinta vermelha. A zona "um" foi à zona central, com 1.0m², e valor de dez pontos: a zona "dois" foi distanciada $2,0 \mathrm{~m}$ do centro do quadrado central e possuía valor de oito pontos: a zona "três" foi dis- tanciada 3,0m do centro do quadrado central com valor de seis pontos e a zona "quatro" foi distanciada a 4,0m do centro do quadrado central, com valor de quatro pontos. As bolas que caíam do outro lado da quadra, porém fora do alvo tinham o valor de três pontos. As bolas que caíam depois da rede fora da quadra de voleibol, tinham o valor de dois pontos e as tentativas em que a bola tocava a rede, a fita superior, as antenas laterais ou caíam antes da rede, tinham o valor de um ponto (Figura 1). A largura das linhas seguiu o padrão da quadra de Voleibol, ou seja, cinco centímetros, e pertencia à zona de maior pontuação. Cada saque foi computado como uma tentativa.

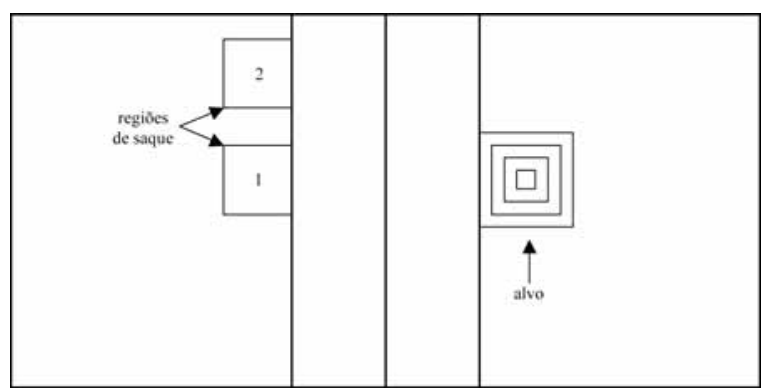

Figura 1. Zona de saque e posição do alvo durante 0 teste de saque de voleibol (adaptado de Teixeira(18)).

Os indivíduos foram divididos de forma randômica em 2 (dois) grupos de igual número em relação à frequência de feedback que foi fornecida: G50\%, que recebia feedback a cada duas execuções e G33\%, que recebia feedback a cada três execuções, baseado no consenso da literatura de que a faixa de frequência de $C R$, que auxilia a aquisição de habilidades motoras, está entre $25 \%$ e $60 \%$. Além disso, optamos também por utilizar uma tarefa que privilegia a validade ecológica dos resultados.

O experimento consistiu de 70 execuções na fase de aquisição e 10 execuções no teste de transferência. O teste de transferência foi aplicado 5 minutos após o término da fase de aquisição, sem o fornecimento de feedback. A fase de aquisição foi realizada em dois dias, sendo que cada sujeito realizou um bloco de 35 tentativas em um dia e outro bloco de 35 tentativas após dois dias. Após cada tentativa, o sujeito recebia outra bola e aguardava a informação fornecida pelo 
experimentador (de acordo com o grupo experimental), com um atraso de 5 segundos após o término da tentativa, e então se preparava para a tentativa seguinte, o que resultou em um intervalo inter-tentativas de 7 segundos. Durante a fase de aquisição, as tentativas foram real izadas na região de saque $1 \mathrm{e}$ durante o teste de transferência, na região de saque 2 (Figura 1).

\section{RESULTADOS}

Os resultados foram descritos em blocos de dez tentativas e o desempenho foi analisado pelo alcance de sua meta, ou seja, a pontuação al cançada no referido saque (escores relativos ao alvo=10, 8, 6, 4, 3, 2, 1). No caso do al cance da meta, utilizou-se à somatória de pontos, a fim de verificar o valor exacto que os sujeitos alcançaram nas tentativas, ou seja, os pontos que eles alcançaram. Essa medida foi utilizada porque é sabido que com a prática um aprendiz apresenta ganhos em precisão, gerando um aumento de sua pontuação.

Dada a natureza "ordinal" da variável dependente e o fato do teste de normalidade não ter mostrado distribuição normal, optou-se pela utilização de testes nãoparamétricos. O desempenho de cada grupo em termos dos pontos al cançados é ilustrado na Figura 2.



Figura 2. Somatória de pontos dos grupos G33\%e G50\%nas duas fases do experimento.

Para fins de análise intra-grupo foi conduzido em cada grupo um teste de Friedman na fase de aquisição e um teste de Wilcoxon entre o último bloco da aquisição e o bloco da transferência. Em termos de comparações entre grupos, analisou-se os desempenhos da fase de aquisição e do último bloco de tenta tivas da fase de aquisição e o bloco do teste de transferência, por meio do teste de U de Mann Whitney.
Concernente ao teste a posteriori, no caso das comparações intra-grupo, na fase de aquisição, utilizouse o o teste de Wilcoxon. Para controle do erro tipo 1 foi utilizado o procedimento sequencial Holm de Bonferroni. Esse procedimento requer a divisão do nível de significância adoptado $(0,05)$ pelo número de comparações realizadas (Ex: na comparação intragrupo, nos pontos, utilizamos 4 blocos, nesse caso, 6 comparações, na comparação entre grupos, utilizamos os 8 grupos, nesse caso, 28 comparações) para localizar a menor diferença. Ao ser localizada, o alfa é dividido pelo número de comparações menos uma e assim sucessivamente, até não poder localizar a diferença.

Com relação à fase de aquisição, na análise intragrupo, pode-se observar na Figura 2 que os grupos mantiveram o desempenho durante toda a fase. Isso foi parcialmente confirmado pelo teste de Friedman, visto que foi detectada diferença significativa para o grupo $G 50 \%\left[\chi^{2}(6, n=10)=14,946, p=0,021\right]$, mas para o $\mathrm{G} 33 \%$ não foi detectada diferença $\left[\chi^{2}(6\right.$, $\mathrm{n}=10)=4,403, \mathrm{p}=0,622]$. Para o grupo $\mathrm{G} 50 \%$, o teste de Wilcoxon, com o procedimento sequencial Holm de Bonferroni, não identificou diferenças significativas, porém quando se analisa sem o procedimento sequencial Holm de Bonferroni, verifica-se a diferença entre os blocos 3 e $6(p=0,008)$ e entre os blocos 6 e $7(p=0,015)$. Isto significa que o grupo melhorou seu desempenho até o bloco 6 , mas piorou o desempenho no bloco 7. Com relação à comparação entre o último bloco da aquisição e a fase de transferência, a Figura 2 indica que o G33\% piorou e o G50\% manteve seu desempenho. Contudo, o teste de Wilcoxon não detectou diferenças significativas para os grupos, todos com $\mathrm{p}>0,05$.

$\mathrm{Na}$ análise entre grupos, na fase de aquisição, podese observar que o G33\% teve um desempenho superior ao G50\% durante toda a fase. Isso foi confirmado pelo teste $U$ de Mann Whitney, visto que foram detectadas diferenças significativas no bloco 2 ( $Z=$ $2,270, G L=6,10,500, p=0,023)$, no bloco $3(Z=-$ $2,421, G L=6,18,000, p=0,015)$ e no bloco 7 ( $Z=-$ $2,990, G L=6,20,000, p=0,002)$. Esses resultados indicam que o G 33\% foi melhor que o G50\% na fase de aquisi ção. Com relação à comparação entre o último bloco da aquisição e a fase de transferência, a analise da Figura 2 indica que o G33\% foi melhor 
que o G50\%. Esse resultado foi confirmado pelo teste $U$ de Mann Whitney, visto que foram detectadas diferenças significativas nos blocos 7 ( $Z=-2,990$, $\mathrm{GL}=1,10,500, \mathrm{p}=0,002$ ) e no bloco da fase de transferência $(Z=-2,349, G L=1,19,000, p=0,0190)$.

\section{DISCUSSÃO E CONCLUSÃO}

O objectivo deste estudo foi investigar os efeitos de diferentes frequências de feedback na aprendizagem de habilidades motoras. Para isso foi utilizado um experimento com fase de aquisição e teste de transferência e duas frequências de $C R$ que têm suporte nos estudos revisados. Além disso, outra opção foi utilizar uma tarefa que privilegia a validade ecológica, para testar se os resultados obtidos em tarefas de laboratório são replicados em situações mais próximas de ensino-aprendizagem.

Primeiramente, por não ter sido utilizado um grupo controle, foi necessário verificar se ocorreu aprendizagem. Os resultados da análise de somatória de pontos, intra-grupo, mostraram aumento nos pontos somente para o G50\%. Esse aumento na somatória dos pontos é um indicativo de aprendizagem, o que permite levar adiante a discussão dos resultados. Levando para uma visão sistémica de aprendizagem motora, é possível observar que o G33\% foi mel hor, pois conseguiu estabelecer mais relações entre o feedback extrínseco e o feedback intrínseco, ou seja, conseguiu trocar energia com o meio ambiente em busca de uma evolução de complexidade. Isto reflectiu-se numa melhora qualitativa, tendo o G33\% conseguido um desempenho superior em relação ao G50\%, demonstrando assim uma melhor adaptação à nova situação imposta.

Os resultados obtidos neste estudo mostraram que, nas fases de aquisição e transferência, o G33\% apresentou superioridade no desempenho em relação ao $\mathrm{G} 50 \%$, o que mostra que o grupo que recebeu uma informação a cada três execuções apresentou um desempenho superior em relação ao grupo que recebeu informação a cada duas execuções. Tais resultados corroboram com os resultados do estudo de Teixeira(17), que mostraram uma tendência de superioridade com uma frequência menor de feedback, bem como os do estudo de Chiviacowsky e Tani(6), que mostraram que a redução da frequência de fedback não provocou resultados negativos para a aprendizagem.
As pesquisas produzidas até então não conseguiram identificar a frequência de feedback que mais favorece a aprendizagem, o que pode ser devido à utilização de diferentes tarefas. Isso é um indicativo de que a quantidade de informação que mais favorece a aprendizagem está relacionada às características da tarefa. Apesar do feedback ainda ser considerado uma variável de grande importância no processo de aprendizagem, não podemos afirmar que as tentativas sem feedback não influenciam a aprendizagem(3). Pelos estudos anteriormente citados, verificamos que mesmo as tentativas sem feedback parecem auxiliar no processo de aprendizagem.

Os achados do presente estudo e de outros de ${ }^{(2,4,8,}$ 19) contradizem as primeiras pesquisas sobre esse tema, de que prover mais feedback é melhor para aprendizagem. Um número reduzido de CR é considerado eficaz para que a aprendizagem ocorra. Alguns estudos $(6,8,19)$ têm mostrado que algumas condições ou variações de CR (amplitude, CR reduzido, resumido, etc.) prejudicam o desempenho durante a fase de aquisição, ou apresentam desempenho similar aos grupos com $100 \%$ de CR, mas mel horam o desempenho quando medido na fase de retenção e transferência.

Esse efeito da menor frequência pode ser explicado pel o facto de $a$ alta frequência de CR pode fazer com que o aprendiz não utilize as informações intrínsecas para a correcção do erro e, como consequência, não desenvolva adequadamente a capacidade de detectálo e corrigi-lo, comportamento observado nos resultados desse estudo. Tais resultados dão suporte à hipótese de orientação(10), de que o CR frequente levaria ao bloqueio do processamento das informações intrínsecas sobre o movimento anteriormente executado, informações essas fundamentais no desenvolvimento do mecanismo de detecção e correcção de erros. Consequentemente, o aprendiz se tornaria dependente da informação fornecida por meio de al gum meio externo e não obteria bom desempenho na ausência dessa informação, o que foi observado nos resultados. A pesar dos resultados indicarem a vantagem de uma menor frequência de CR para o saque do voleibol, ainda fica a questão da interacção entre frequência de CR e a complexidade da tarefa, sendo essa uma importante questão a ser investigada futuramente. 
Por último, os resultados do presente estudo também vão ao encontro dos obtidos em estudos que utilizaram tarefas laboratoriais(1, 2, 5, 20, 17, 19, 21, 23) e indicaram superioridade dos grupos que receberam informação reduzida, ou seja, CR menor que $100 \%$. Esse panorama dos resultados também conduz a proposta de verificar se os mesmos resultados são replicados com outras formas de reduzir a quantidade de informação, tais como o fedback sumário e a amplitude de feedback.

\section{CORRESPONDÊNCIA}

\section{Prof. Dr. U mberto César Corrêa}

Universidade de São Paulo

Escola de Educação Física e Esporte

Departamento de Pedagogia do Movimento

do Corpo Humano

Laboratório de Comportamento Motor

Av. Mello Moraes, 65 - Cidade Universitária

CEP 05508-900 - São Paulo, SP

E-mail: ivanwall@usp.br 
REFERÊNCIAS BIBLIOGRÁFICAS

1. Anderson DI., Magill RA, Sekiya H (2001). Motor Learning as a Function of KR Schedule and Characteristics of Task-Intrinsic Feedback. Journal of M otor Behavior 33: 5966.

2. Baird IS, Hughes GH (1972). Effects of frequency and specificity of information feedback on acquisition and extinction of a positioning task. Perceptual and M otor Skills 34: 567-572.

3. Bilodeau EA, Bilodeau IM (1958). Variable frequency of knowledge of results and the learning of a simple skill. Journal of Experimental Psychology 55: 379-383.

4. Chalmers A (1993). A fabricação da ciência. São Paulo, Unesp Editora.

5. Chiviacowsky S, Tani G (1993). Efeitos da Freqüência de Conhecimento de Resultados na Aprendizagem de uma Habilidade Motora em Crianças. R evista Paulista de Educação Física 7: 45-57.

6. Chiviacowsky S, Tani G (1997). Efeitos da Freqüência de Conhecimento de Resultados na Aprendizagem de Diferentes Programas Motores Generalizados. R evista Paulista de Educação Física 11: 15-26.

7. Clark SC (2005). Freqüência de Conhecimento de Resultados e Aprendizagem Motora: Iinhas atuais de pesquisa e perspectivas: In: Tani G (Ed.) Comportamento $M$ otor: A prendizagem e D esenvolvimento. Guanabara Koogan, 185-204.

8. Coca AA, Tertuliano IW, Pereira FAS, Ugrinowitsch H., Gimenez R (2002). Amplitude de feedback como um fator de incerteza na aquisição de habilidades motoras. In: A nais do III Seminário de Comportamento M otor, Gramado.

9. Corrêa UC (2006). Estrutura e organização da prática na aprendizagem motora: uma análise das pesquisas com tarefas “do mundo real”. In: Rigolin da Silva LR (Ed.) Desempenho esportivo: treinamento com crianças e adolescência. São Paulo: Manole (in press).

10. Salmoni AW, Schmidt RA, Walter CB (1984). Knowledge of results and motor learning: a review and critical reappraisal. Psychological Bulletin 95: 355-386.

11. Schmidt RA (1975). A schema theory of discrete motor skill learning. Psychological Review 82: 225-260.

12. Schmidt RA (1993). Aprendizagem e performance motora: dos princípios à prática. Movimento, São Paulo.

13. Schmidt RA, Wrisgerg CA (2001). A prendizagem e Performance M otora: U ma abordagem da aprendizagem baseada no problema. Artmed, Porto Alegre, 2o Edição.
14. Tani G (1989). Significado, Detecção e Correção do erro de Performance no processo Ensino-Aprendizagem de Habilidades Motoras. Revista Brasileira de Ciências \& M ovimento 3: 50-58.

15. Tani G, Dantas LEPBT, Manoel EJ (2005). EnsinoAprendizagem de habilidades motoras: um campo de pesquisa de síntese e integração de conhecimentos. In: Tani G (Ed.) Comportamento Motor: Aprendizagem e Desenvolvimento. Guanabara Koogan, 106-115.

16. Tani G, Meira Júnior CM, Gomes FRF (2005). Freqüência, precisão e localização temporal de conhecimento de resultados e o processo adaptativo na aquisição de uma habilidade motora de controle da força manual. R evista Portuguesa de Ciências do D esporto 5: 59-68.

17. Teixeira LA (1993). Freqüência de Conhecimento de Resultados na Aquisição de Habilidades Motoras: efeitos transitórios e de aprendizagem. Revista Paulista de Educação Física 7: 8-16.

18. Ugrinowitsch H, Manoel EJ (1999). Interferência contextual: manipulação de parâmetros e programas na aquisição da habilidade "saque" do voleibol. R evista Paulista de Educação Física 13: 197-216.

19. Ugrinowitsch $\mathrm{H}$, Tertuliano IW, Coca AA, Pereira FAZ, Gimenez R (2003). Freqüência de feedback como um fator de incerteza na aprendizagem de uma tarefa de preensão. Revista Brasileira de Ciência e M ovimento 11: 41-47.

20. Weeks DL, Kordus R (1998). Relative Frequency of knowledge of performance and motor skill learning. R esearch Q uarterly for Exercise and Sport 69: 224-230.

21. Wisntein CJ, Schmidt RA (1990). Reduced frequency of knowledge of results enhances motor skill learning. Journal of Experimental Psychology Learning, M emory and Cognition 16: 667-691.

22. Wrisberg CA, Wulf G (1997). Diminishing the effects of reduced frequency of knowledge of results on generalized motor program learning. Journal of M otor Behavior 29: 1726.

23. Wulf G, Schmidt RA (1989). Feedback induced variability and the learning of generalized motor programs. Journal of M otor Behavior 26: 348-361.

24. Wulf G, Lee TD , Schmidt RA (1994). Reducing knowledge of results about relative versus absolute timing: differential effects on learning. Journal of $M$ otor Behavior 26 : 362-369. 